\title{
Methylation of Epstein-Barr Virus-Associated Gastric Cancer Suppressor Genes
}

\author{
Tingting Chen ${ }^{1, *}$, Donghui Tian², Yurong Zhang ${ }^{3}$ \\ ${ }^{1}$ Department of Gastroenterology, Binzhou People's Hospital, Binzhou City, P. R. China \\ ${ }^{2}$ Department of Neurology, Binzhou People's Hospital, Binzhou City, P. R. China \\ ${ }^{3}$ Department of Hepatobiliary Surgery, Binzhou People's Hospital, Binzhou City, P. R. China \\ Email address: \\ TingtingChen2019@163.com (Tingting Chen) \\ *Corresponding author
}

\section{To cite this article:}

Tingting Chen, Donghui Tian, Yurong Zhang. Methylation of Epstein-Barr Virus-Associated Gastric Cancer Suppressor Genes. American Journal of Clinical and Experimental Medicine. Vol. 7, No. 2, 2019, pp. 54-60. doi: 10.11648/j.ajcem.20190702.13

Received: May 29, 2019; Accepted: July 25, 2019; Published: July 29, 2019

\begin{abstract}
Objective: To identify the methylation silenced tumor suppressor genes in Epstein-Barr virus (EBV)-associated gastric cancer (EBVaGC). Methods: EBV-positive (GT38, PT and SNU719) and negative (SGC7901) gastric cancer cell lines were selected and treated with 5-Aza-CdR. Then real-time fluorescence quantitative PCR was used to validate the results of microarray, and methylation-specific PCR (MSP) and bisulfite genomic sequencing (BGS) were adopted to detect the CpG island methylation levels of gene promoters. Results: The expression levels of 6 differentially expressed genes (H19, LOXL1, ARMCX2, LXN, CDH3 and MMP7) before and after 5-Aza-CdR treatment were confirmed by real-time qPCR, which are consistent with the results of microarray analysis. There were different degrees of methylation in LOXL1 gene promoter in EBVaGC. GT38 and PT were fully methylated, and SGC7901 and HGC-27 was unmethylated, suggesting that this gene is a candidate methylation silenced tumor suppressor gene. The methylation rate of LOXL1 in EBVaGC was significantly higher than that in EBV-negative gastric cancer (EBVnGC). Conclusion: The promoter region of candidate tumor suppressor gene LOXL1 shows high methylation status, indicating that EBV critically accounts for the methylation of LOXL1 gene regulatory region. EBV is involved in the pathogensis of EBVaGC that aberrant methylation occurred in promoter CpG island, which inactivates tumor suppressor genes.
\end{abstract}

Keywords: Epstein-Barr Virus, Gastric Cancer, Methylation, Tumor Suppressor Gene

\section{Introduction}

Epstein-Barr virus (EBV), the strain of which was successfully established by Epstein and Barr for the first time in 1964 by the in vitro suspension culture of Burkitt African children lymphoma cells, leads to a variety of malignancies (e.g. nasopharyngeal carcinoma) [1]. EBV mainly infects B lymphocytes manifested with non-proliferation infection [2], and stratified squamous epithelial cells accompanied by viral replication inducing $\mathrm{B}$ cell-derived tumors and epithelial cell-derived tumors [3]. The disease is widely distributed worldwide and may be epidemic because more than $90 \%$ of healthy adults are carrying this virus [4]. The onset ages range mostly between 15 and 30 years old, and children below 6 often show inapparent infection. EBV-related diseases occur throughout the year, which seems to peak in late autumn and early winter. Once infected, the patients usually get relatively long-lasting immunity. The relationship between EBV infection and gastric cancer was confirmed in the 1990s. The EBV infection rate of lymphoepithelioma-like gastric cancer is up to $80 \%$ to $100 \%$, and there that of ordinary gastric cancer differs regionally by about $10 \%$ [5]. DNA methylation is the most cutting-edge epigenetic mechanism, in which the role of methylation-based epigenetic change in tumorigenesis is of the greatest concern. In addition to mutation and deletion, the hypermethylation of tumor suppressor gene promoter is considered to dominate the inactivation of tumor suppressor gene, which plays an important role in the occurrence and development of tumor [6]. About $20 \%$ of human tumors are related to viral infections. The abnormal epigenetics of host 
cell caused by virus infections has been spotlighted in the current research on the carcinogenic mechanism of tumor virus [7].

Studies have shown that hypermethylation of some tumor-related genes [8] (tumor suppressor gene, apoptosis-related gene and mismatch repair gene, etc.) exists in EBV-associated gastric cancer (EBVaGC), whose methylation frequency and methylation density of promoter $\mathrm{CpG}$ island are higher than those of EBV-negative gastric cancer (EBVnGC). High methylation is negatively correlated with the expression of tumor suppressor genes in EBVaGC, such as p16 and p73. EBV infection induces aberrant methylation in $\mathrm{CpG}$ island that inactivates cancer suppressor genes, whch may be the mechanism of EBV involved in the occurrence of EBVaGC. However, the relationship between EBV genome methylation and host cell gene methylation, as well as how EBV induces the methylation of suppression cancer genes of host cell have not been clarified yet [9].

EBV-positive and-negative gastric cancer cell lines were selected as the object in this study, which were treated with 5-Aza-CdR [10] to induce the re-expression of methylation silenced tumor suppressor genes, and an untreated control group was established simultaneously. The methylation statuses of target genes in EBVaGC and EBVnGC were detected and compared, and the relationship between the methylation of cancer suppressor genes and clinical-pathological characteristics were further analyzed and identified define EBVaGC-specific apparent molecular genetic marker.

\section{Materials and Methods}

\subsection{Apparatus}

The apparatus included PTC-100 PCR, ABI-7000 quantitative fluorescence PCR (ABI), spectrophotometer, high-speed low-temperature centrifuge (Eppendor), $\mathrm{CO}_{2}$ incubator (SHEIL-LAB), G2545A hybrid reactor, G2565BA microarray scanner (Agilent), inverted microscope, glass tube (NarishigeGD-1 core tube, $1 \times 90 \mathrm{~mm}$ core-free tube), gel imaging system, ultra0low-temperature refrigerator, and constant-temperature water bath.

\subsection{Reagents and Materials}

EBV cells were stored in our laboratory. DMEM culture medium, TRIzol and fetal bovine serum were purchased from
GIBCO. 5-aza-deoxycytidine was purchased from Sigma. Microarray hybridization reagents were bought from Agilent. The other reagents and materials include AM8436 aaUTP (Alnbion), $0.2 \mathrm{ml}$ PCR reaction tube, $15 \mathrm{ml}$ centrifuge tube, 1 $\mathrm{ml}$ and $1.5 \mathrm{ml}$ Eppendor tubes, PCR reagents (dNTp, $\mathrm{MgCl}_{2}$, $10 \times$ buffer), trypsin, agarose and DNA marker DL-2000.

\subsection{Cell Culture and Treatment}

DMEM medium was used to cultivate 4 cell lines (GT38, PT, SNU719 and SGC7901), subcultured once every 2 to 3 days. $5 \mathrm{mg}$ was then taken and fully dissolved with DMEM medium, allowing the constant volume to $43.8 \mathrm{~mL}$, which was prepared into a $500 \mu \mathrm{mol} / \mathrm{L}$ mother liquor and stored at $-20^{\circ} \mathrm{C}$. Then it was diluted to a $10 \mu \mathrm{mol} / \mathrm{L}$ working solution by DMEM medium. The cells in logarithmic growth phase were sampled. When the cell convergence concentration reached $70 \%--80 \%$, the medium was abandoned and the cells were washed twice with PBS. DMEM medium of 5-Aza-CdR containing $10 \mu \mathrm{mol} / \mathrm{L}$ methylation inhibitor was added, and the group without drug was used as the control. The total cellular RNA was extracted after 4 continous days.

\subsection{Differentially Expressed Genes Identified by Real-Time qPCR}

The total cellular RNA was extracted for RNA inverse transcription according to the standard conditions required by reverse transcription kit. cDNA obtained from inverse transcription was used as the template for PCR reaction and stored at $-20^{\circ} \mathrm{C}$. Amplification and data analysis were conducted on a LightCycler480 II fluorescent quantitative PCR instrument with the SYBR Green I dye method. The result data of Real- time qPCR were analyzed to determine the $\mathrm{Ct}$ value of each group. $\mathrm{Ct}$ value represents the number of cycles experienced when fluorescence signal in each reaction tube reached the specified threshold. Ct value of each template is linearly correlated with the logarithm of initial copy number of the template. $\Delta \mathrm{Ct}$ value of each sample was analyzed with the relative quantitative method and $\Delta \mathrm{Ct}=$ target gene $\mathrm{Ct}$ internal reference gene $\mathrm{Ct} .2^{-\Delta \Delta} \mathrm{Ct}$ was calculated according to $\Delta \Delta \mathrm{Ct}=$ treatment group $\Delta \mathrm{Ct}$ - control group $\Delta \mathrm{Ct}$. When the amplification efficiencies of target and internal reference genes are close, $2^{-\Delta \Delta}{ }^{\mathrm{Ct}}$ represents the times of expression of target gene in the sample in the treatment group relative to that in the control group (Table 1).

Table 1. Primer sequences of real-time $q P C R$ detection.

\begin{tabular}{|c|c|c|c|c|}
\hline Gene & Type & Sequence & Annealing temperature $\left({ }^{\circ} \mathrm{C}\right)$ & Length (bp) \\
\hline \multirow[t]{2}{*}{ H19 } & Primer 1 & GGCCTTCCTGAACACCTTAG & \multirow[t]{2}{*}{56} & \multirow[t]{2}{*}{142} \\
\hline & Primer 2 & TGAGCTGGGTAGCACCATTT & & \\
\hline \multirow[t]{2}{*}{ ARMCX2 } & Primer 1 & CTGCTTGTCAATTCCATTGC & \multirow[t]{2}{*}{58} & \multirow[t]{2}{*}{142} \\
\hline & Primer 2 & CTGGCACTTGGGTACTGAGA & & \\
\hline \multirow[t]{2}{*}{ LOXL1 } & Primer 1 & ACTGCCAGTGGATCGACATA & \multirow[t]{2}{*}{59} & \multirow[t]{2}{*}{121} \\
\hline & Primer 2 & ATGTTGCATCTAACCACGTT & & \\
\hline LXN & Primer 1 & GGAGATTCCCTGGCAAA & 58 & 81 \\
\hline \multirow[t]{2}{*}{ MMP7 } & Primer 1 & GACATCATGATTGGCTTTGC & \multirow[t]{2}{*}{58} & \multirow[t]{2}{*}{128} \\
\hline & Primer 2 & TCCTCATCGAAGTGAGCATC & & \\
\hline
\end{tabular}




\begin{tabular}{lllll}
\hline Gene & Type & Sequence & Annealing temperature $\left({ }^{\circ} \mathbf{C}\right)$ & Length $(\mathbf{b p})$ \\
\hline CDH3 & Primer 1 & AGATCACCATCTGCAACCAA & 146 \\
& Primer 2 & GTCACCTTCCTCGTTGACCT & 59 & \\
\multirow{2}{*}{ GAPDH } & Primer 1 & CCTAGACACACCATGGGGAAGGTGA & & 450 \\
& Primer 2 & ATGATCTTGAGGCTGTTGTCATA & & \\
\hline
\end{tabular}

\subsection{DNA Methylation}

Fresh gastric cancer and corresponding pericarcinomatous tissues resected from 975 gastric cancer patients in our hospital were collected. The tumor was taken immediately after being separated from the body under aseptic conditions. A tissue block with the size of $1 \mathrm{~cm}^{3}$ was taken from the non-necrotic tumor tissue, placed in a sterile bottle and treated as soon as possible or stored at $-80^{\circ} \mathrm{C}$. Tissue DNA was routinely extracted with the phenol-chloroform-isoamyl alcohol method. Meanwhile, part of tissues were taken from each sample, fixed with PBS-10\% formalin and embedded with paraffin to prepare tissue sections for pathological diagnosis and EBER in-situ hybridization to screen EBVaGC. 23 cases of EBVaGC sample with strong EBER in-situ hybridization signal and high quality DNA were selected as the positive EBV group and 22 cases of EBVnGC sample were rendered as the negative ENV group.

Nucleic acid was extracted from fresh gastric cancer tissue and corresponding pericarcinomatous tissue cells, and the extracted DNA was modified and purified by nitrate. MSP was conducted on EBV positive gastric cancer cell lines modified by utilizing hydrosulfite, EBV negative gastric cancer cell lines and tissue DNA as the templates. The methylation-specific primers, non-methylation-specific primers and the sizes of MSP products are shown in Table 2. To further detect the specific methylation status at $\mathrm{CpG}$ locus in the promoter region of LOXLI coding gene and verify the MSP results, primer line BGS sequences outside MSP primer sequence were designed online using MethPrimer. The results were recorded by a UV gel imaging system.

Table 2. Methylation-specific PCR and sulfite salt-gene sequencing primers.

\begin{tabular}{llllll}
\hline Gene & Type & Sequence & Site & Annealing temperature $\left({ }^{\circ} \mathbf{C}\right)$ & Length $($ bp) \\
\hline \multirow{6}{*}{ LOXL1 } & M1 & TTAGTAGGCGGATTAGGAGTC & $-43 \sim 135$ & \multirow{2}{*}{60} & 178 \\
& M2 & ACGATACCCAAAACTACACGAA & & & \\
& U1 & AGTAGGTGGATTAGGAGGTTGA & $-41 \sim 137$ & 58 & \\
& U2 & AAACAATACCCAAAACTACACAAA & $-126 \sim-101$ & \multirow{2}{*}{60} & 374 \\
& 1 & TTTTAGGGATTGAGGGAGTATTGTA & $223 \sim 248$ & & \\
\hline
\end{tabular}

\subsection{Statistical Analysis}

The gene promoter methylation detection data were analyzed by PPMS1.5. The measurement data were compared by the calibration test. $\mathrm{P}=0.5$ was considered statistically significant.

\section{Results}

\subsection{Differentially Expressed Genes Identified by Real-Time qPCR}

To verify the differential genes identified by gene chip analysis and preliminarily screen methylation silenced genes,
6 differentially expressed genes (H19, LOXL1, ARMCX2, LXN, CDH3 and MMP7) were selected for real-time qPCR. The results of real-time qPCR show that the 6 genes above in three EBV positive gastric cancer cell lines treated with Aza had different degrees of expression up-regulation. However, 5 genes (LOXL1, ARMCX2, LXN, CDH3 and MMP7) in EBV negative gastric cell line SGC7901 have undifferentiated expression before and after Aza treatment, which is consistent with the result of gene chip. Only the expression of $\mathrm{H} 19$ gene up-regulated after treating EBV negative gastric cell line SGC7901, which is different from the result of gene chip detection. The comparisons between real-time qPCR and fold change values of chip detection are summarized in Table 3.

Table 3. Real-time $q P C R$ and microarray detection fold change values.

\begin{tabular}{lllllllll}
\hline \multirow{2}{*}{ Gene } & GT38 & & PT & & SUN719 & \multicolumn{3}{c}{ SCG7910 } \\
\cline { 2 - 9 } & qPCR & chip & qPCR & chip & qPCR & chip & qPCR & chip \\
\hline H19 & 6.32 & 18.17 & 3.25 & 9.15 & 4.76 & 24.50 & 3.24 & -1.02 \\
LOXL1 & 7.89 & 8.66 & 17.27 & 8.75 & 13.45 & 11.22 & -1.19 & -1.28 \\
ARMCX2 & 5.06 & 15.64 & 3.41 & 12.54 & 7.11 & 17.87 & -1.07 & -1.32 \\
LXN & 3.72 & 3.38 & 3.25 & 4.65 & 4.92 & 4.34 & 1.15 & 1.05 \\
CDH3 & 6.50 & 3.10 & 4.92 & 2.29 & 14.93 & 2.20 & 1.23 & 1.10 \\
MMP7 & 3.42 & 9.99 & 2.83 & 4.26 & 3.03 & 5.95 & 1.15 & 1.22 \\
\hline
\end{tabular}

\subsection{Candidate Tumor Suppressor Genes Promoter Methylation Detection}

The MSP results show that LOXL1 gene promoter was highly methylated in GT38 and PT cell lines. Methylation and non-methylation were simultaneously detected in most cell lines, but methylation was not found in SGC7901 and HGC-27 cell lines. The results are exhibited in Figure 1. 


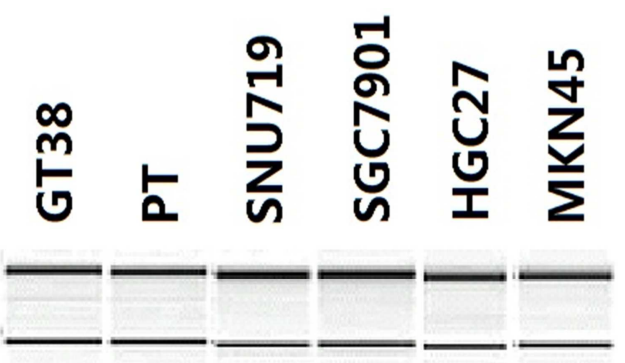

Figure 1. Electrophoresis results of LOXL1 gene prompter methylation.
In the 23 cases of EBVaGC tissue, two MSP types were detected in LOXLI coding gene promoter, including 12 cases of type $\mathrm{M}(12 / 23,54.5 \%)$ and 11 cases of type M+U (11/23, $45.5 \%)$. Two MSP types were detected in the 23 cases of EBVaGC pericarcinomatous tissue, including 1 case of type $\mathrm{M}+\mathrm{U}(1 / 23,4.3 \%)$ and 22 cases of type $\mathrm{U}(22 / 23,95.7 \%)$. In the 22 cases of EBVnGC tissue, two MSP types were detected in LOXLI coding gene promoter, including 15 cases of type $\mathrm{M}+\mathrm{U}(15 / 22,68.2 \%)$ and 5 cases of type $\mathrm{U}(7 / 22,31.8 \%)$. Two MSP types were detected in the 22 cases of EBVnGC tissue, including 2 case of type $\mathrm{M}+\mathrm{U}(2 / 22,9.1 \%)$ and 20 cases of type U (20/22, 90.9\%). The MSP detections of LOXLI coding gene promoter in EBVaGC and EBVnGC tissues are shown in Figure 2.

A

EBVaGC

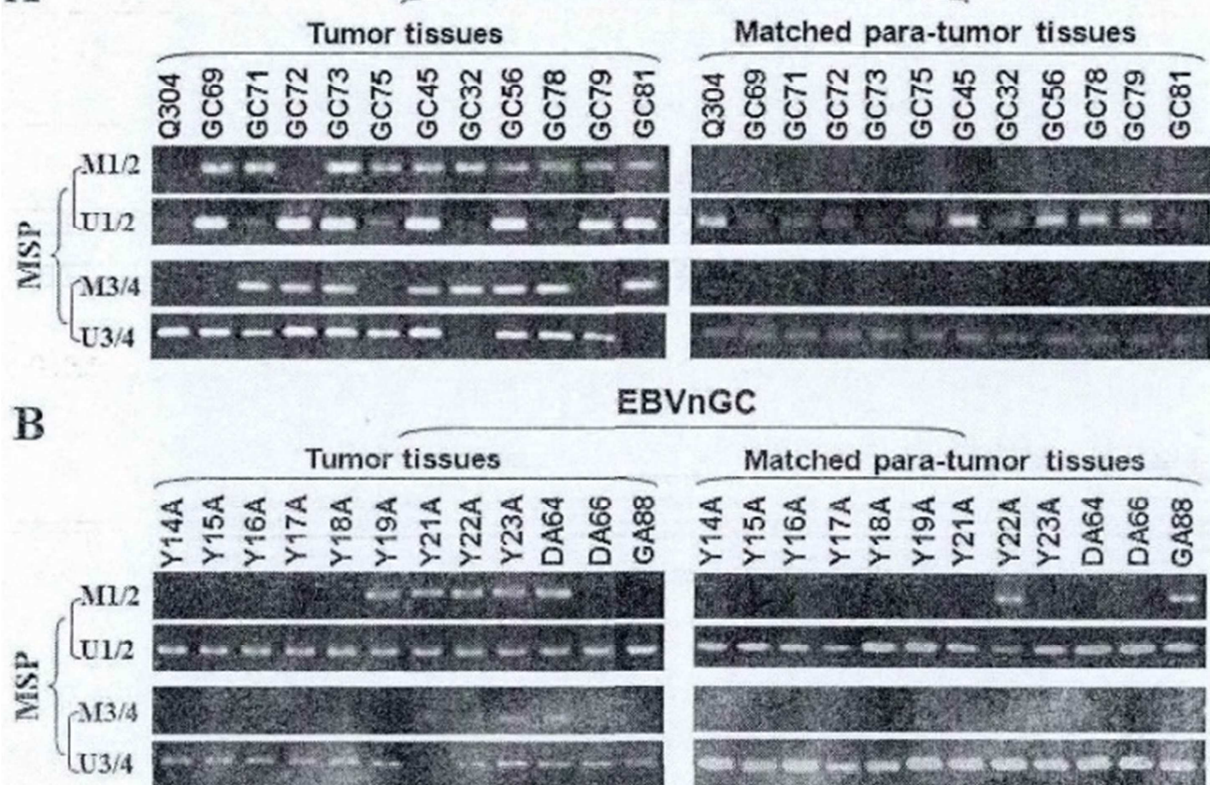

Figure 2. Electrophoresis results of EBVaGC and EBVnGCLOXL1 gene prompter methylation.

BGS detected the methylation status of LOXL1 gene promoter of some samples. The sequencing results prove that in the 5 cases of EBV positive sample (GT38, PT, Akata, GC89 and GC95) with type M MSP result, most sites at CpG site in LOXL1 promoter region were methylated. In the 4 cases of Ebv positive samples (C666, GC98, GC100 and GC81) with type $\mathrm{M}+\mathrm{U}$ MSP result, $\mathrm{CpG}$ site in LOXL1 promoter region was partially methylated. In the 2 cases of samples (SGC7901 and HGC27) with type U MSP result, methylation did not occur at $\mathrm{CpG}$ site in LOXL1 promoter region. Moreover, in the 3 cases of EBVaGC tissue (GC98,
GC100 and GC81), some CPG sites of LOXL1 coding gene had two different methylated forms, i.e. methylation (C) and non-methylation ( $\mathrm{T}$ ) existed simultaneously.

In EBV related and negative gastric cancer tissues, the positive methylation rate of LOXL1 gene promoter in ENV negative gastric cancer tissue $(68.2 \%, 15 / 22)$ was significantly higher than that of pericarcinomatous tissue. The positive rate $(100 \%, 23 / 23)$ of gastric cancer methylation was significantly higher than that of $\operatorname{EBV}\left(\chi^{2}=6.41\right.$ (correction), $\left.\mathrm{P}<0.05\right)$. The results are shown in Table 4.

Table 4. LOXL1 gene methylation rates.

\begin{tabular}{llll}
\hline Group & Positive (case) & Negative (case) & Methylation rate $(\chi \%)$ \\
\hline EBVaGC $(\mathrm{n}=23)$ & 23 & 0 & $100 \%$ \\
EBVaGC matched para-carcinoma $(\mathrm{n}=23)$ & 1 & 22 & $4.3 \%$ \\
EBVnGC $(\mathrm{n}=23)$ & 15 & 7 & $68.2 \%$ \\
EBVnGC matched para-carcinoma $(\mathrm{n}=23)$ & 2 & 20 & $9.1 \%$ \\
\hline
\end{tabular}

EBVaGC vs. EBVaGC matched para-carcinoma, $\chi^{2}=42.17, P<0.01$; EBVnGC vs. EBVnGC matched para-carcinoma, $\chi^{2}=16.20, P<0.01 ; \mathrm{EBVaGC}$ vs. EBVnGC, $\chi^{2}=6.41, P<0.05$. 


\subsection{Relationship Between LOXL1 Gene Methylation and Clinical Pathological Characteristics}

LOXL1 gene methylation in gastric cancer tissue and the gender, age, pathological histology type, diseased site, lymphatic metastasis and EBV infection of patients were compared (Table 5). The result show that LOXL1 gene methylation was not significantly correlated with the gender, age, pathological histology type, diseased site and morbidity of lymphatic metastasis. Only the methylation rates differed significantly $\left(\chi^{2}=6.41\right.$ (correction), $\left.P<0.05\right)$.

Table 5. Relationship between LOXL1 gene methylation and the clinical pathological characteristics of gastric cancer patients.

\begin{tabular}{|c|c|c|c|c|c|c|c|}
\hline Item & & Case No. & Positive & Negative & Positive rate (\%) & $\chi^{2}$ & $P$ value \\
\hline \multirow{2}{*}{ Gender } & Male & 39 & 32 & 7 & 82.1 & \multirow{2}{*}{1.28} & \multirow{2}{*}{$>0.05$} \\
\hline & Female & 6 & 6 & 0 & 100 & & \\
\hline \multirow{2}{*}{ Age } & $<50$ & 11 & 10 & 1 & 90.9 & \multirow{2}{*}{0.46} & \multirow{2}{*}{$>0.05$} \\
\hline & $\geq 50$ & 34 & 28 & 6 & 82.4 & & \\
\hline \multirow{2}{*}{$\begin{array}{l}\text { Pathological } \\
\text { type }\end{array}$} & Highly and mediumly differentiated adenocarcinoma & 9 & 8 & 1 & 88.9 & \multirow{2}{*}{0.17} & \multirow{2}{*}{$>0.05$} \\
\hline & Lowly differentiated adenocarcinoma & 36 & 30 & 6 & 83.3 & & \\
\hline \multirow{4}{*}{ Site } & Gastric cardia & 4 & 4 & 0 & 100 & \multirow{4}{*}{2.37} & \multirow{4}{*}{$>0.05$} \\
\hline & Gastric antrum & 20 & 16 & 4 & 80.0 & & \\
\hline & More than 2 sites & 10 & 9 & 1 & 90.0 & & \\
\hline & Gastric stump & 3 & 3 & 0 & 100 & & \\
\hline \multirow{4}{*}{$\begin{array}{l}\text { Lymphatic } \\
\text { metastasis } \\
\text { EBV } \\
\text { infection* }\end{array}$} & Yes & 28 & 24 & 4 & 85.7 & \multirow{2}{*}{0.09} & \multirow{2}{*}{$>0.05$} \\
\hline & No & 17 & 14 & 3 & 82.4 & & \\
\hline & EBVaGC & 23 & 23 & 0 & 100 & 641 & $<005$ \\
\hline & EBVnGC & 22 & 15 & 7 & 68.2 & 6.41 & $<0.05$ \\
\hline
\end{tabular}

*EBVaGC and EBVnGC, $\chi 2=6.41, \mathrm{P}<0.05$.

\section{Discussion}

EBV infection is closely associated with the occurrence of some gastric cancers. The results of in-situ hybridization showed that EBV could be detected in almost all tumor cells in EBVaGC tissues, indicating that EBV infection occurred in the early gastric carcinogenesis and tumor originated from the monoclonal growth of EBV infected cells [11]. Further studies have shown that compared with EBVnGC, EBVaGC has unique clinical pathological features. For example, the onset of EBVaGc presents a low-aging tendency, and the diseased part is commonly in the gastric body or cardia region at gastric fundus. The pathological type is mainly tubular adenocarcinoma or poorly differentiated adenocarcinoma. In terms of histopathological type, diffuse gastric cancer occurs more frequently than intestinal gastric cancer with low lymphatic metastasis rate and desirable prognosis [12]. The expression of virus coding gene of EBVaGc is not identical to that of EBV-related tumors such as NPC and Burkitt's lymphoma. For instance, recognized EBV cell transformation gene, i.e. latent membrane protein (LMP) 1 coding gene, is not expressed in EbVaGC [13]. Its differences from EBVnGC and other EBV-related tumors suggest that EBVaGC has a unique formation mechanism [14].

DNA methylation is the most cutting-edge epigenetic mechanism that has been extensively accessed, in which the role of methylation-based epigenetic change in tumorigenesis is of the greatest concern. In addition to mutation and deletion, the hypermethylation of tumor suppressor gene promoter is considered to determine the functional inactivation of tumor suppressor gene, which plays an important role in tumorigenesis and tumor evolution [15]. Currently, studies on EBVaGC methylation have been focusing on the detection of methylation status of known genes at DNA level, while methylation silenced cancer suppressor genes caused by EBV infection are rarely screened and identified. [16]. DNA methylation is not involved in the change of DNA sequence, therefore this change is reversible, suggesting that the hypermethylation status in gene promoter region can be eliminated by demethylation to reactivate silent genes, especially tumor suppressor ones [17]. Nucleoid methyltransferase inhibitors represented by 5-Aza-CdR have been widely used to reverse the aberrant methylation of tumor cells, induce the re-expression of methylation silenced tumor suppressor genes and inhibit the growth of tumor cells, thus killing tumor cells [18]. EBV positive and negative gastric cancer cell lines were selected in this study as the object and treated with 5-Aza-CdR to re-express methylation silenced tumor suppressor genes. In the meantime, an untreated control group was established, the total cellular RNA of the experimental and control groups was prepared, and then the gene expression level was detected by high-throughput whole genome microarray expression profile chip to screen the silent cancer suppressor genes due to methylation. Then the methylation of these genes was verified and analyzed to identify methylation silenced cancer suppressor genes specific to EBV positive gastric cancer cell lines. Hence, the methylation status of target genes in EBVaGC and EBVnGC tissues was further detected and compared to analyze the relationship between the methylation of identified cancer suppressor genes and clinical pathological features so as to clarify the action mechanism of EBV infection in EBV positive cell lines and $\mathrm{EBVaGC}$ tissue methylating candidate cancer suppressor genes, and to define EBVaGC specific apparent molecular genetic marker.

Agilent gene expression profile [19] experiment: a 
comparative analysis was conducted on the differences of gene expression between 5-Aza-CdR treatment group and non-treatment group of EBV negative and positive gastric cancer cell lines. According to the different degrees of gene expression and SAS system and functional clues provided by relevant literatures, 6 differentially expressed genes (H19, LOXL1, ARMCX2, LXN, CDH3 and MMP7) were selected for real-time fluorescence quantitative PCR analysis to verify the results of gene chip [20]. The results show that 6 genes in three EBV positive gastric cancer cell lines with Aza treatment had different degrees of expression upregulation. Nevertheless, 5 genes (LOXL1, ARMCX2, LXN, CDH3 and MMP7) in EBV negative gastric cell line SGC7901 had undifferentiated expressions before and after Aza treatment, which is consistent with the result of gene chip. Only H19 gene had expression upregulation in EBV negative gastric cell line SGC7901 after treatment, which was different from the result of gene chip detection. The results above show that real-time fluorescence quantitative PCR accords with chip detection result, proving the reliability of gene chip result to some extent and indicating that hypermethylation of these genes is caused by EBV infection, i.e. silent genes involved by EBV may be related to the occurrence of EBV-related gastric cancers [21].

This study found that LOXL1 gene promoter was hypermethylated in EBV-related gastric cancer tissues, indicating that DNA methylation status in LOXL1 gene regulation region is an essential regulatory mechanism of gene expression [22]. The MSP results show that LOXL-1 promoter was fully methylated in EBV positive gastric cancer cell lines GT38 and PT, partially methylated in EBV positive cell lines (B95-8, Raji, GT39 and SNU719), and unmethylated in EBV negative gastric cancer cell lines SGC7901 and HGC27. The methylation rate of LOXL-1 promoter in EBV-related gastric cancer tissues was significantly higher than that in EBV negative gastric cancer tissues. The results above reveal that the LOXL-1 gene methylation in EBVaGC tissues was highly correlated to EBV infection, and LOXL-1 gene was the candidate methylation silenced cancer suppressor gene of EBVaGC [23].

DNMT introduces an activated methyl group into DNA chain with SAM as the donor and forms $\mathrm{m} 5 \mathrm{C}$ through covalent binding with carbon potential of genome $\mathrm{CpG}$ binuclear general acid [24]. Methylation transferases include maintenance methylation transferase (DNMTI) and re-methylase (DNMT3a, DNMT3b). In the form of maintenance extensive methylation, these three enzymes have both overlapped and respective unique functions [25]. EBV is latently infected in tumor tissues. In EB-related tumor tissues such as Burkitt lymphoma and NPC, EBV genome is hypermethylated and close to the expression of nuclear antigen (EBNA) 2 6 genes and rise phase genes through methylation of $\mathrm{Wp}$ and $\mathrm{Cp}$ promoter genes and rise phase gene promoters $\mathrm{Zp}$ and $\mathrm{Rp}$, thus avoiding the killing effect of cytotoxicity T cell on EBV infected cells [26]. Some studies also hold that virus and host cells interact in EBV carcinogenesis [27]. EBV can induce host cell gene to aberrant methylation while developing self-defense via its own gene methylation, which results in cell cycle disorder and cell transformation, as well as promotes the occurrence and development of EBV-related tumors epigenetically. This study shows that EBVaGc methylation frequency and methylation density of promoter $\mathrm{CpG}$ island were much higher than those of EBVnGC, and hypermethylation was negatively correlated with the expression of cancer suppressor genes in EBVaGc tissues such as p16 and p73. EBV infection induced aberrant methylation in $\mathrm{CpG}$ island, thereby inactivating cancer suppressor genes. It might be a mechanism of EBV being involved in the occurrence of EBVaGC. However, it is still undefined how EBV induces the methylation of host cell cancer suppressor gene. LMP1 can interact with cell cycle regulatory gene through multiple mechanisms and thus promote cell proliferation and suppress cell apoptosis. LMP2 and LMP1 are commonly distributed on cell membrane with synergistic effects, but the specific function remains unclear yet. LMP2A might be related to transmembrane signaling. In EBV-infected B cells, LMP2A can block the $\mathrm{Ca}^{2+}$ passage of $\mathrm{B}$ cells and suppress virus entering the lytic cycle, thus maintaining the latency infection of EBV. However, the function in epithelial cells has not been determined [28].

The study above indicates that the expression of virus-associated genes up-regulated that of DNMTs by relevant signaling pathways, and finally induced aberrant methylation in $\mathrm{CpG}$ island, thus resulting in the inactivation of cancer suppressor genes. In EBV positive cell line and EBVaGC tissue, LOXL1 methylation degree was significantly higher than those of EBV negative cell line and EBVnGC tissue, inferring that EBV accounts for the DNA methylation in LOXL1 gene regulatory region and that LOXL1 gene might be the candidate methylation silenced cancer suppressor gene specific to EBVaGC.

In this study, EBV positive (PT, GT38 and SNU719) and negative (SGC7901) gastric cancer cell lines cultivated in vitro were treated with a demethylation reagent 5 -Aza-CdR to extract the total cellular RNA before and after treatment. The results of gene chip were verified by real-time fluorescence quantitative $\mathrm{PCR}$, and $\mathrm{CpG}$ island methylation in some gene promoter regions was detected by methylation specificity PCR (MSP) and hydrosulphite genome sequencing method (BGS). The gene (LOXL1) with reported tumor suppressor function was selected as the candidate cancer suppressor gene. In EBV positive cell line and EBVaGC tissue, LOXL1 gene promoter region was hypermethylated and its methylation degree was significantly higher than those of EBV negative cell lines and EBVnGC tissue. Promoter $\mathrm{CpG}$ island had aberrant methylation and thus inactivated cancer suppressor gene.

\section{Conclusion}

In conclusion, the hypermethylation of cancer suppressor gene promoter governed the functional inactivation of cancer suppressor gene in addition to mutation and deficiency. EBV infection induced aberrant methylation of $\mathrm{CpG}$ island, which thus inactivated cancer suppressor gene. It might be a mechanism of EBV being involved in the occurrence of EBVaGC. 


\section{References}

[1] Young KH, Zhang D, Malik JT, Williams EC. Fulminant EBV-driven CD8 T-cell lymphoproliferative disorder following primary acute EBV infection: a unique spectrum of T-cell malignancy. Int J Clin Exp Pathol 2008, 1: 185-197.

[2] Katsumura KR, Maruo S, Takada K. EBV lytic infection enhances transformation of B-lymphocytes infected with EBV in the presence of T-lymphocytes. J Med Virol 2012, 84: 504-510.

[3] Shamaa AA, Zyada MM, Wagner M, Awad SS, Osman MM, Abdel Azeem AA. The significance of Epstein Barr virus (EBV) \& DNA topoisomerase II alpha (DNA-Topo II alpha) immunoreactivity in normal oral mucosa, oral epithelial dysplasia (OED) and oral squamous cell carcinoma (OSCC). Diagn Pathol 2008, 3: 45.

[4] Long HM, Leese AM, Chagoury OL, Connerty SR, Quarcoopome J, Quinn LL, et al. Cytotoxic CD4+ T cell responses to EBV contrast with CD8 responses in breadth of lytic cycle antigen choice and in lytic cycle recognition. J Immunol 2011, 187: 92-101.

[5] Lee SM, Oh YL, Kim KM, Ko YH, Lee J. Coexistence of EBV associated nasopharyngeal undifferentiated carcinoma and gastric lymphoepithelioma-like carcinoma. Pathology 2010, 42: 684-686.

[6] Rusiecki JA, Al-Nabhani M, Tarantini L, Chen L, Baccarelli A, Al-Moundhri MS. Global DNA methylation and tumor suppressor gene promoter methylation and gastric cancer risk in an Omani Arab population. Epigenomics 2011, 3: 417-429.

[7] Li L, Su X, Choi GC, Cao Y, Ambinder RF, Tao Q. Methylation profiling of Epstein-Barr virus immediate-early gene promoters, BZLF1 and BRLF1 in tumors of epithelial, NK- and B-cell origins. BMC Cancer 2012, 12: 125.

[8] Zhao C, Bu X. Promoter methylation of tumor-related genes in gastric carcinogenesis. Histol Histopathol 2012, 27: 1271-1282.

[9] Grafodatskaya D, Choufani S, Ferreira JC, Butcher DT, Lou Y, Zhao $\mathrm{C}$, et al. EBV transformation and cell culturing destabilizes DNA methylation in human lymphoblastoid cell lines. Genomics 2010, 95: 73-83.

[10] Jin Q, Liu C, Yan C, Tao B, Li Z, Cai Z. 5-aza-CdR induces the demethylation of Syk promoter in nasopharyngeal carcinoma cell. Gene 2012, 511: 224-226.

[11] Liu X, Gao Y, Luo B, Zhao Y. Construction and Antiapoptosis Activities of Recombinant Adenoviral Expression Vector Carrying EBV Latent Membrane Protein 2A. Gastroenterol Res Pract 2011, 2011: 182832.

[12] Chen JN, He D, Tang F, Shao CK. Epstein-Barr virus-associated gastric carcinoma: a newly defined entity. J Clin Gastroenterol 2012, 46: 262-271.

[13] Bengtson P, Zetterberg H, Mellberg T, Pahlsson P, Larson G. Characterization of EBV-transformed B-cells established from an individual homozygously mutated (G329A) in the FUT7 alpha1,3-fucosyltransferase gene. Scand J Immunol 2005, 62: 251-258.

[14] Seo JS, Jun SM, Kwon SW, Oh IH, Kim TG, Lee SK. Establishment and characterization of gastric carcinoma cell clones expressing LMP2A of Epstein-Barr virus. Int J Mol Med 2010, 25: 11-16.

[15] Lee JH, Kim SH, Han SH, An JS, Lee ES, Kim YS. Clinicopathological and molecular characteristics of Epstein-Barr virus-associated gastric carcinoma: a meta-analysis. J Gastroenterol Hepatol 2009, 24: 354-365.

[16] Sun Y, Jiang X, Xu Y, Ayrapetov MK, Moreau LA, Whetstine $\mathrm{JR}$, et al. Histone $\mathrm{H} 3$ methylation links DNA damage detection to activation of the tumour suppressor Tip60. Nat Cell Biol 2009, 11: 1376-1382.

[17] Ren JZ, Huo JR. [Effect of 5-Aza-CdR on expression and methylation of E-cadherin gene in human colon carcinoma cells]. Chin J Cancer 2010, 29: 38-42.

[18] Painter HJ, Altenhofen LM, Kafsack BF, Llinas M. Whole-genome analysis of Plasmodium spp. Utilizing a new agilent technologies DNA microarray platform. Methods Mol Biol 2013, 923: 213-219.

[19] Naher L, Kiyoshima T, Kobayashi I, Wada H, Nagata K, Fujiwara $\mathrm{H}$, et al. STAT3 signal transduction through interleukin-22 in oral squamous cell carcinoma. Int $\mathrm{J}$ Oncol 2012, 41: 1577-1586.

[20] Zhao J, Jin H, Cheung KF, Tong JH, Zhang S, Go MY, et al. Zinc finger E-box binding factor 1 plays a central role in regulating Epstein-Barr virus (EBV) latent-lytic switch and acts as a therapeutic target in EBV-associated gastric cancer. Cancer 2012, 118: 924-936.

[21] Bell A, Bell D, Weber RS, El-Naggar AK. CpG island methylation profiling in human salivary gland adenoid cystic carcinoma. Cancer 2011, 117: 2898-2909.

[22] Liu X, Tang X, Zhang S, Wang Y, Wang X, Zhao C, et al. Methylation and Expression of Retinoblastoma and Transforming Growth Factor-betal Genes in Epstein-Barr Virus-Associated and -Negative Gastric Carcinomas. Gastroenterol Res Pract 2012, 2012: 906017.

[23] Sahin M, Sahin E, Gumuslu S, Erdogan A, Gultekin M. DNA methylation or histone modification status in metastasis and angiogenesis-related genes: a new hypothesis on usage of DNMT inhibitors and S-adenosylmethionine for genome stability. Cancer Metastasis Rev 2010, 29: 655-676.

[24] Gravina GL, Marampon F, Piccolella M, Motta M, Ventura L, Pomante R, et al. Hormonal therapy promotes hormone-resistant phenotype by increasing DNMT activity and expression in prostate cancer models. Endocrinology 2011, 152: 4550-4561.

[25] Park SY, Kook MC, Kim YW, Cho NY, Kim TY, Kang GH. Mixed-type gastric cancer and its association with high-frequency $\mathrm{CpG}$ island hypermethylation. Virchows Arch 2010, 456: 625-633.

[26] Perera RA, Samaranayake LP, Tsang CS. Shedding dynamics of Epstein-Barr virus: A type 1 carcinogen. Arch Oral Biol 2010, 55: 639-647.

[27] Engels N, Yigit G, Emmerich CH, Czesnik D, Schild D, Wienands J. Epstein-Barr virus LMP2A signaling in statu nascendi mimics a $\mathrm{B}$ cell antigen receptor-like activation signal. Cell Commun Signal 2012, 10: 9.

[28] Ushiku T, Chong JM, Uozaki H, Hino R, Chang MS, Sudo M, et al. p73 gene promoter methylation in Epstein-Barr virus-associated gastric carcinoma. Int J Cancer 2007, 120: 60-66. 